\title{
Factors influencing brightness and beam quality of conventional and distributed Bragg reflector tapered laser diodes in absence of self-heating
}

\author{
Simeon Newton Kaunga-Nyirenda ${ }^{1}$, Stephen Bull' ${ }^{1}$ Jun Jun Lim ${ }^{1}$, Karl-Heinz Hasler ${ }^{2}$, \\ Jörg Fricke ${ }^{2}$, Eric C. Larkins ${ }^{1}$ \\ ${ }^{1}$ Photonic and Radio Frequency Engineering Group (PRFEG), Electrical Systems and Optics Research Division, Faculty of \\ Engineering, University of Nottingham, University Park, Nottingham, NG7 2RD, UK \\ ${ }^{2}$ Leibniz-Institut für Höchstfrequenztechnik, Ferdinand-Braun-Institut, Gustav-Kirchhoff-Str. 4, Berlin 12489, Germany \\ E-mail: simeon.kaunga-nyirenda@nottingham.ac.uk
}

Abstract: In this study, the authors examine some of the factors affecting the brightness and the beam quality of high-power tapered lasers. The large volume resonators required to achieve a high-power, high-brightness operation make the beam quality sensitive to carrier lensing and a multimode operation. These cause bleaching of the regions outside the ridge waveguide. The beam quality in the conventional and the distributed Bragg reflector tapered lasers is examined in the absence

30 of the self-heating effects to investigate the effect of the carrier lensing effects. The influence of the front facet reflectivity and the taper angle on the beam quality is investigated. The beam quality was found to degrade with an increase in the front facet reflectivity and for the larger taper angles in the conventional tapered lasers, especially at low ridge waveguide currents. Finally, the performance of the conventional tapered lasers employing a beamspoiler was assessed. The beam quality was 35 found to be comparable with that achieved in the DBR tapered lasers.

\section{Introduction}

40

Over recent years, high-brightness laser diode technology has progressed rapidly in response to competitive and evolving markets and diode lasers have found new applications in, for example, medicine and materials processing [1]. For 45 many applications of interest, the key design objective for the high-power laser diodes is to maximise the output power while maintaining good beam quality and reliability. The simplest way to achieve high power with high efficiency is to use a large resonator such as in a broad-area 50 (BA) laser. However, this type of laser suffers from poor beam quality because of the excitation of the higher modes and beam filamentation [2]. The brightness of the BA lasers is sensitive to the thermal- and the carrier-induced lensing effects. Narrow-stripe ridge waveguide (RW) lasers can be 55 designed to operate in a single lateral mode [3]. However, the output power is limited by the small gain volume of the device. Tapered laser diodes [4-9] offer a solution that combines the advantages of the BA-lasers, in terms of the high-power output, with the good beam quality offered by 60 the narrow-stripe RW lasers. For many applications, the tapered laser diodes are found to offer an economical solution because of their simplicity and compatibility with the current fabrication techniques $[5,6]$.

A tapered laser diode consists of an RW section coupled to

65 a tapered amplifier section. The straight RW acts as a mode selective filter, which rejects any higher order lateral modes that are fed back from the tapered gain section. Careful design of the RW section ensures that the taper section is only excited by the fundamental mode. The taper angle is chosen so that the forward propagating wave laterally expands in the taper section to fill the output aperture of the taper, whereas experiencing amplification because of the gain of the pumped laser medium. The expansion of the beam over a larger facet width lowers the power density at the facet. This helps to overcome the gain saturation because of the spatial hole burning and minimises the risk of device failure, which allows the realisation of the high output powers while maintaining good beam quality. The operation of a tapered laser diode is intrinsically dependent on a good RW filter performance. For optimal performance, the mismatch between the RW and the tapered sections must be minimised and the mode-filtering performance of the RW must be optimised.

In this paper, some of the factors that affect the brightness of the high-power tapered lasers in the absence of self-heating are studied. In particular, the effects of the front facet reflectivity and the taper angle on the conventional and the distributed Bragg reflector (DBR) tapered lasers are examined. The effect of these factors on the beam quality and the RW performance are studied by using a metric that quantifies the filtering performance of the RW [10]. The effect of introducing a beamspoiler to improve the beam quality is also assessed. The paper is organised as follows. In Section 2, the laser structure investigated in this paper is 


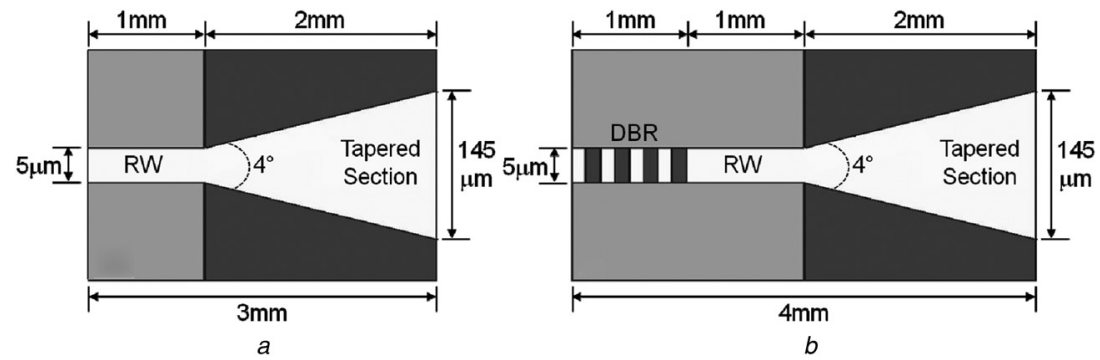

Fig. 1 Geometrical structure of the $1060 \mathrm{~nm}$ tapered laser

$a$ Conventional tapered laser

$b$ DBR tapered laser

presented. A description of the electrical and the optical models employed in our in-house 2.5D laser simulator is bandstructure and the gain model used and their calibration to the experimental measurements is also presented in this section. The beam quality of the conventional tapered lasers is discussed and compared with that of the DBR tapered lasers in Section 4. The influence of the front facet reflectivity and the taper angle on the beam quality is considered in Section 5. The improvements obtained by using a beamspoiler in the conventional lasers [11] is then discussed and compared with the performance of a standard DBR laser.

\section{Device structure}

The laser structure investigated is a $1060 \mathrm{~nm}$ laser with a triple InGaAs/GaAsP QW with a vertical waveguide width of $4.8 \mu \mathrm{m}$ [12]. The conventional tapered laser diode (Fig. 1a) is comprised of an RW section $(1 \mathrm{~mm})$ and a tapered section $(2 \mathrm{~mm})$. The DBR tapered laser includes a DBR $(1 \mathrm{~mm})$ at the end of the RW section (Fig. 1b). The standard tapered laser has a taper angle of $4^{\circ}$ and an output aperture of $\sim 145 \mu \mathrm{m}$. The front and the rear facet reflectivities are 1 and $31 \%$, respectively [12]. In the DBR tapered laser, the rear facet reflectivity is represented as an effective reflectivity of $31 \%$ in the RW and $0.01 \%$ outside the RW. The RW and the tapered sections can be independently biased. This structure has a small vertical beam divergence of $15^{\circ}$ (FWHM) and a low internal loss of $0.9 \mathrm{~cm}^{-1}$. The RW was index guided and the taper was gain guided with a shallow isolation implant.

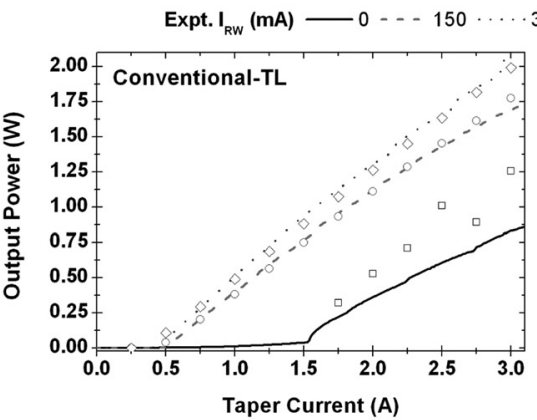

\section{Semiconductor laser model}

The simulations were performed with our in-house $2.5 \mathrm{D} \mathrm{CW}$ multi-wavelength laser simulator [13], comprised of a bipolar electro-thermal model and an optical model, which are solved self-consistently, following an accelerated Fox-Li iterative approach. The optical model is based on the two-dimensional (2D) wide-angle finite-difference beam propagation method and is coupled to the $2 \mathrm{D}$ transverse electrical model via stimulated emission/absorption, spontaneous emission and carrier/temperature induced changes in the complex index. The electrothermal model calculates the carrier density profile for a series of $2 \mathrm{D}$ transverse slices along the laser cavity and includes the drift-diffusion transport and the capture/escape processes between the bound and the unbound states of the QW(s). The basic semiconductor device equations that describe the electronic behaviour of the semiconductor laser have been described in a previous publication [14], including Poisson's equation and the continuity equations for both the bulk and the confined electrons and holes. All the relevant recombination processes (e.g. Shockley-Read-Hall, Auger, spontaneous emission and stimulated emission recombination) are included in the continuity equations. The QW gain was calculated by using a parabolic conduction band and a $4 \times 4$ band $\mathbf{k} \cdot \mathbf{p}$ model for the valence band. The QW index changes were calculated from the Kramers-Kronig transformations of the gain spectra.

The lattice heat equation and the self-heating effects have been ignored, as the main focus of this paper was to investigate the beam evolution and the carrier-induced lensing effects in tapered lasers, without the added complexity of the thermal-lensing effects. However, the

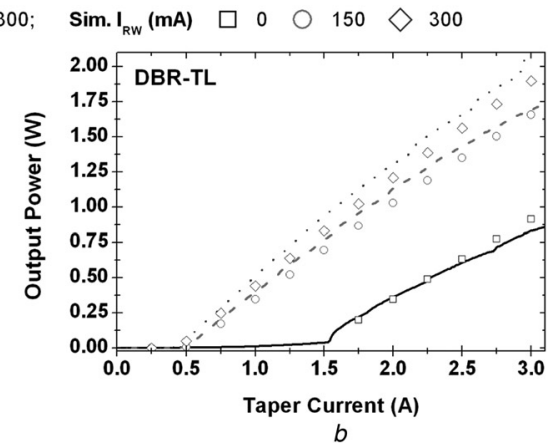

Fig. 2 Experimental and simulated light-current characteristics as a function of the taper current, for different $R W$ currents for $a$ Conventional tapered laser and $b$ DBR tapered laser 
265 temperature dependencies of all the important material parameters are included in order to accurately model the temperature characteristics of the device. Therefore, in all the simulations reported in this paper, the bias currents considered were well below the current levels at which the 270 onset of a thermal rollover is observed. The effect of the thermal effects on the beam quality and its evolution is currently being investigated and will be the subject of a future paper.

The DBR tapered laser includes a passive DBR section at the end of the RW section. The passive DBR serves to fix the emission wavelength and is found to be stable with an RW current within $130 \mathrm{pm}$ [12]. In all the simulations in this paper, a single wavelength (fixed at $1060 \mathrm{~nm}$ ) was simulated because of the good wavelength stability with 280 respect to the current. The DBR section is passive (i.e. unpumped); hence its effect is represented by using a patterned reflectivity within our in-house laser simulator. The effective reflectivity of the DBR was $31 \%$. The reflectivity profile, with a value of $31 \%$ within the RW 285 region and AR-coated at $0.01 \%$ outside the RW, was used at the rear facet to simulate the effect of the DBR.

The simulations of the laser structures in Fig. 1 have been calibrated to, and validated against the experiment in the previous paper, as described in [13]. Good agreement was obtained for the power-current-voltage characteristics, the near- and the far-field patterns, the astigmatism, the beam quality factor $M^{2}$ (based on the $1 / e^{2}$ level) and the emission spectrum (and their evolution with the bias current) [12, 13]. Fig. 2 shows the simulated and the experimental power-current characteristics for both (a) the conventional and (b) the DBR tapered lasers for different RW currents.

\section{Beam quality of the conventional and the DBR tapered lasers}

Simulations were performed on the split-contact $1060 \mathrm{~nm}$ tapered laser for a range of RW and taper currents to investigate the beam quality of both the conventional and the DBR tapered lasers. Fig. 3 shows the simulated near(a, d) and the far-field (b, e) patterns for both the conventional $(\mathrm{a}-\mathrm{c})$ and the DBR $(\mathrm{d}-\mathrm{f})$ tapered lasers for three RW currents at a fixed taper current of $3 \mathrm{~A}$.

The near- and the far-field patterns of the conventional tapered laser show significant sidelobes in the beam profile, as seen in Figs. $3 a$ and $b$. These sidelobes are more pronounced at low RW currents. This indicates that the fundamental and the undesired higher-order lateral modes are reflected at the rear facet. The higher-order modes are excited in the taper region and amplified, resulting in the significant undesired sidelobes observed in the near-field
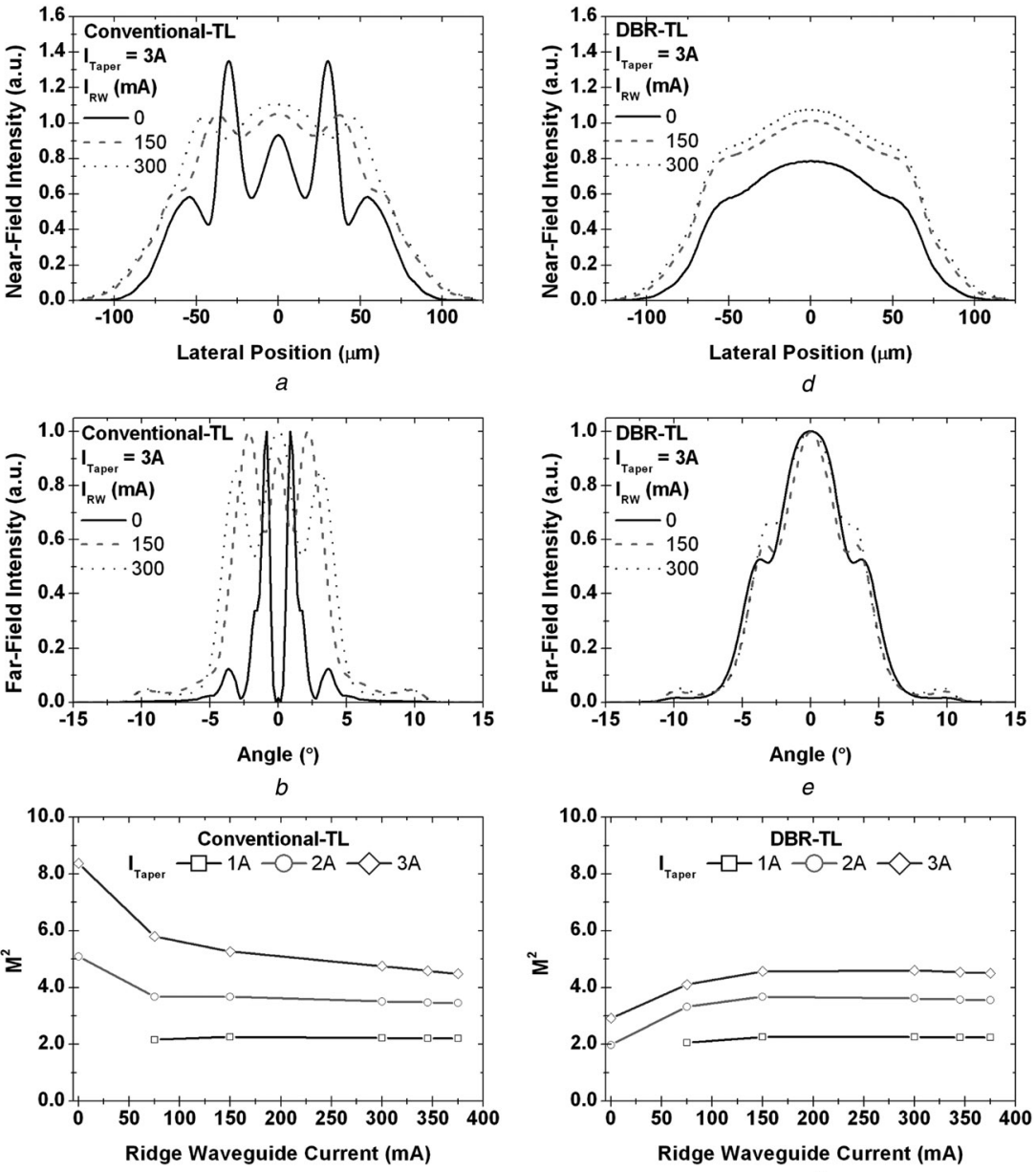

Fig. 3 Simulated near

$a-c$ Results for the conventional and

$d-f$ the DBR tapered laser

Near- (a, d) and far-fields (b, e) for the different RW currents and a taper current of 3 A; evolution of the $M^{2}$ (e, f) with the RW current for taper currents of 1,2 and 
pattern. As the RW current increases, the gain in the RW also increases, so that it provides a significant fraction of the total round-trip gain. As a result, the RW carries a larger proportion of the light and the power outside the RW correspondingly decreases. This also results in reduced absorption bleaching outside the RW. Thus, as the RW current increases, the intensity of the sidelobes is reduced. This filamentation is not observed in the near- and the far-field patterns of the Decause of the good modal filtering by the DBR. The DBR only reflects the light inside the RW, which is amplified and expands gradually in the taper region, leading into a smooth single lobed near-field pattern, as seen in Fig. $3 d$, and consequently a single lobed far-field, as shown in Fig. $3 e$. Figs. $3 c$ and $f$ compare the beam quality of the conventional and the DBR lasers, that is, the evolution of the $M^{2}$ (calculated by the second moment method-ISO 11146 standard) as a function of the RW current for taper currents

415 of 1,2 and $3 \mathrm{~A}$, respectively. The relatively high $M^{2}$ values, compared with the values based on the $1 / e^{2}$ definition, are most likely because of the non-Gaussian distribution of the far-field profile. The beam quality of the conventional tapered lasers degrades with decreasing RW current and is particularly poor when the taper current is high and the RW current is low (Fig. 3c). (Note that the device does not achieve lasing at a taper current of $1 \mathrm{~A}$ and an RW current of $0 \mathrm{~mA}$ ). The degradation of the beam quality at a low RW current and a high taper current is due to the poor RW mode filtering performance in the conventional tapered lasers. The low optical gain in the RW means that the proportion of the optical power is smaller inside the RW. Consequently, the fraction of the optical power outside the RW increases, leading to an increase in absorption bleaching in the regions adjacent to the RW. This allows the unguided higher-order modes to compete for a taper gain, causing an increase in the intensity of the optical sidelobes, and consequently poor beam quality. The combined effect of the DBR and the RW mode filter in the DBR tapered lasers leads to a significant improvement in the beam quality. The $M^{2}$ is less than 4.5 for the bias currents considered (Fig. $3 f$ ). In contrast to the conventional tapered lasers, there is an improvement in the beam quality at low RW currents, even at high taper currents (e.g. at an RW current of $0 \mathrm{~mA}$ and a taper current of $3 \mathrm{~A}, M^{2}>8$ for the conventional tapered lasers compared with $\sim 3$ for the DBR tapered lasers). The superior beam quality at low RW currents and high taper currents, where the conventional tapered lasers struggle, is useful in applications that require a high modulation efficiency and a high output power, for example, display and optical wireless applications. The high modulation efficiency can be obtained by applying a small modulation bias to the RW, whereas applying a high fixed taper current to achieve a high-power output.

A further understanding of the beam quality in the two types of tapered lasers can be obtained by examining the beam evolution inside the laser cavity. Figs. 4 and 5 show the photon distribution in both the conventional and the DBR tapered lasers at RW currents of 0 and $300 \mathrm{~mA}$,
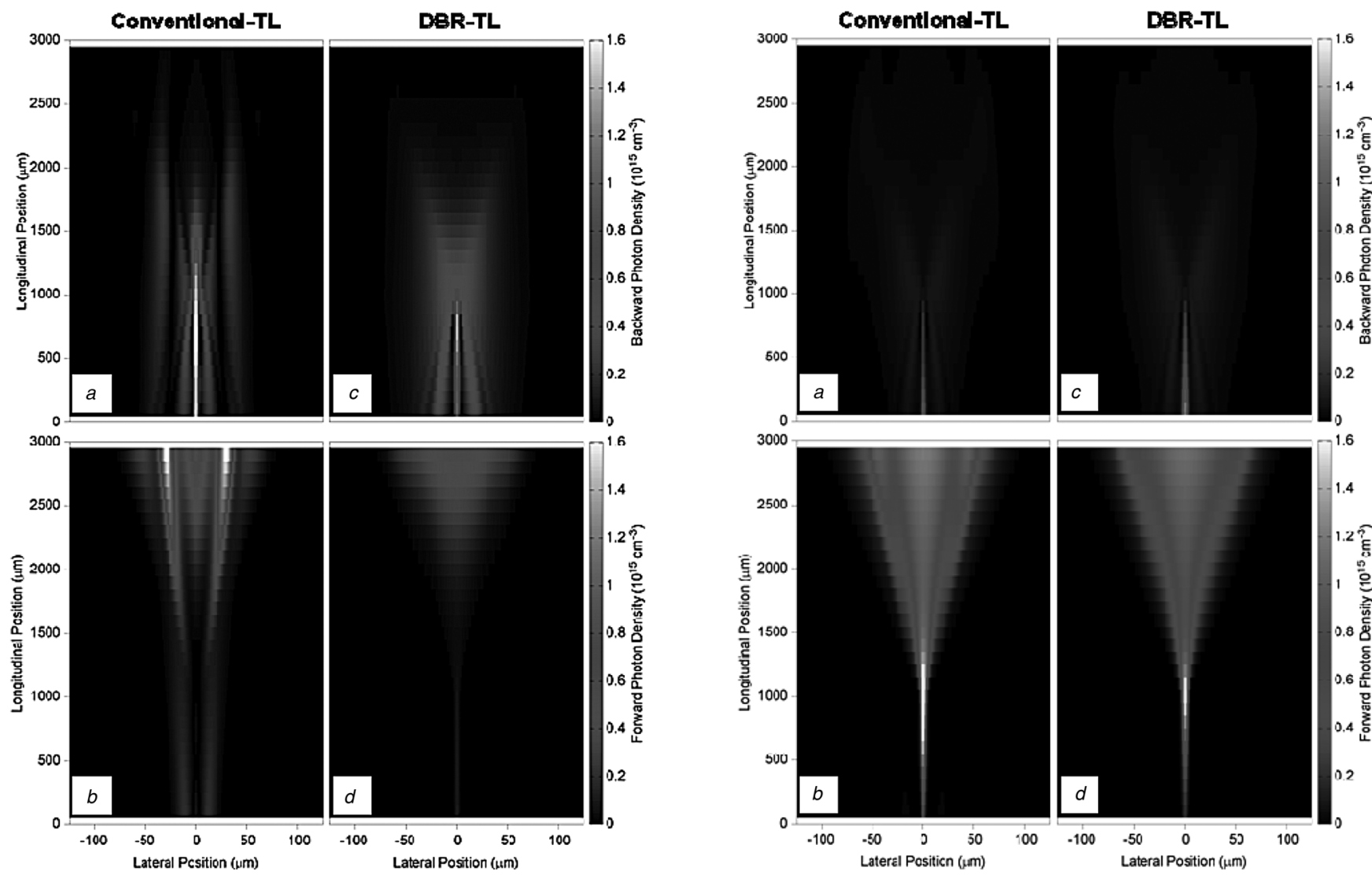

Fig. 4 Photon distribution

Propagating photon distributions for a taper current of 3 A and a RW current of $0 \mathrm{~mA}$ for the conventional $(\mathrm{a}, \mathrm{b})$ and the DBR $(\mathrm{c}, \mathrm{d})$ tapered lasers

Fig. 5 Photon distribution

a, $c$ Backward

$b, d$ Forward

Propagating photon distributions for a taper current of 3 A and an RW current of $300 \mathrm{~mA}$ for the conventional $(\mathrm{a}, \mathrm{b})$ and the $\operatorname{DBR}(\mathrm{c}, \mathrm{d})$ tapered lasers 
respectively, and at a fixed taper current of $3 \mathrm{~A}$. The presence of strong unguided modes outside the RW is evident in the backward propagating fields in the conventional tapered laser at $0 \mathrm{~mA}$ (Figs. $4 a$ and $b$ ). The resulting sidelobes (Figs. $3 a$ and $b$ ) are responsible for the degradation in the beam quality (Fig. 3c). The absence of these sidelobes in

535 the DBR laser (Figs. $4 c$ and $d$ ) results in the much cleaner near- and far-fields (Figs. $3 d$ and $e$ ) and the improved beam quality (Fig. $3 f$ ). The effect of the DBR decreases at high RW currents (Fig. 5), resulting in comparable beam quality for the conventional and the DBR tapered lasers at high

540 RW currents.

The mode filtering performance of the DBR can be observed clearly by considering the backward and the forward propagating optical fields at the rear facet. Fig. 6 shows the backward and the forward photon distributions at the rear three RW currents and a fixed taper current of $3 \mathrm{~A}$. Higher-order modes are present in the backward propagating optical fields in both the conventional (Fig. 6a) and the DBR (Fig. 6c) tapered lasers. At the RW-taper interface, part of

550 the backward travelling wave couples into the RW, whereas the rest is coupled into the higher-order modes. The amplitude of the higher-order modes increases as the RW current decreases (Figs. $6 a$ and $c$ ). At the rear facet in the DBR laser, the DBR only reflects the wave inside the RW, 5 whereas the undesired fields outside the RW are not reflected back into the laser cavity (Fig. 6d). However, in the conventional tapered laser, the unguided fields outside the RW are reflected back into the cavity (Fig. 6b), which leads to the sidelobes observed in the near- and the far-field patterns.

\section{Factors influencing beam quality}

Further simulations were performed to investigate the various 565 factors that affect the beam quality in the high-power tapered laser diodes. In particular, the influences of the front facet reflectivity and the taper geometry on the beam quality were investigated.

\subsection{Influence of the front facet reflectivity}

The beam quality of the tapered lasers has been observed to be influenced by the level of carrier bleaching outside the $\mathrm{RW}$, which is itself influenced by the level of pumping of the RW section, for a given taper current. At a fixed RW and taper current, the level of carrier bleaching outside the RW can be influenced by the strength of the backward travelling optical fields, which depends on the front facet reflectivity. Fig. 7 shows the evolution of the $M^{2}$ as function of the RW current for the fixed taper currents $(1,2$ and $3 \mathrm{~A}$ ) for the facet reflectivities of 0.01 and $1 \%$ on both the conventional and the DBR tapered lasers. The beam quality in the conventional tapered laser degrades significantly as the front facet reflectivity increases (Figs. $7 a$ and $b$ ), because of increased carrier bleaching caused by significant back reflections from the front facet. This degradation is most severe at the low RW currents and higher taper currents in the conventional tapered lasers $\left(M^{2}\right.$ $>6$ at an RW current of $0 \mathrm{~mA}$, for taper currents greater than 2 A). The beam quality is largely unaffected by the increase in the front facet reflectivity in the DBR lasers (Figs. $7 c$ and $d$ ). The DBR-RW mode filter suppresses the higher-order lateral modes and the filamentation even when significant back reflections are present from the output facet. The level of carrier bleaching and hence the strength of the sidelobes that are responsible for the degradation of the beam quality are therefore largely independent of the front facet reflectivity for the cases investigated in the DBR tapered laser.

A further insight into the effect of the front facet reflectivity on the beam quality can be obtained by quantifying the spatial
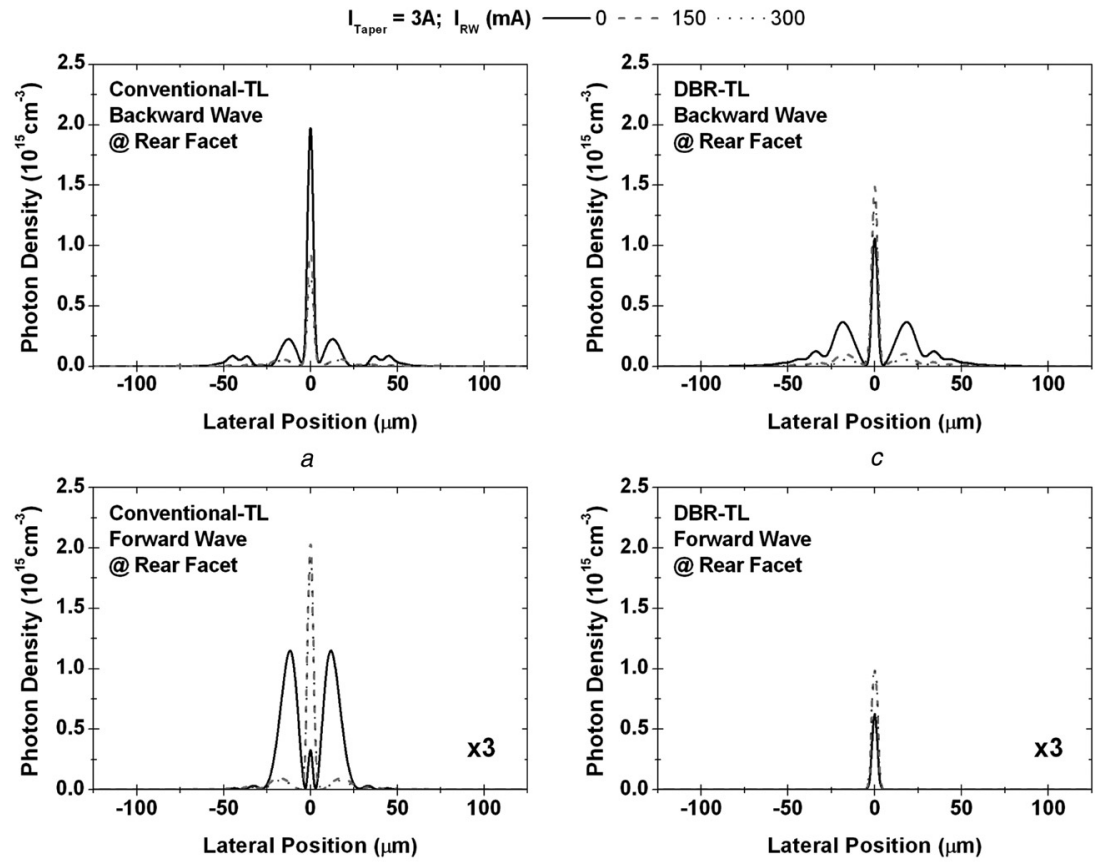

$b$

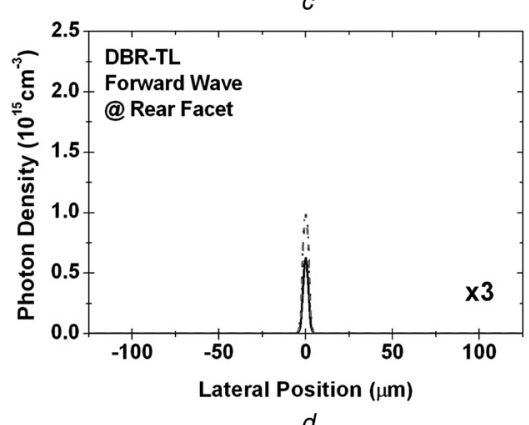

Fig. 6 Backward and the forward photon distributions

a, $c$ Backward

$b, d$ Forward

Propagating photon distributions, for the different RW currents and a taper current of $3 \mathrm{~A}$, at the rear facet of the conventional (a, b) and the DBR (c, d) tapered lasers 

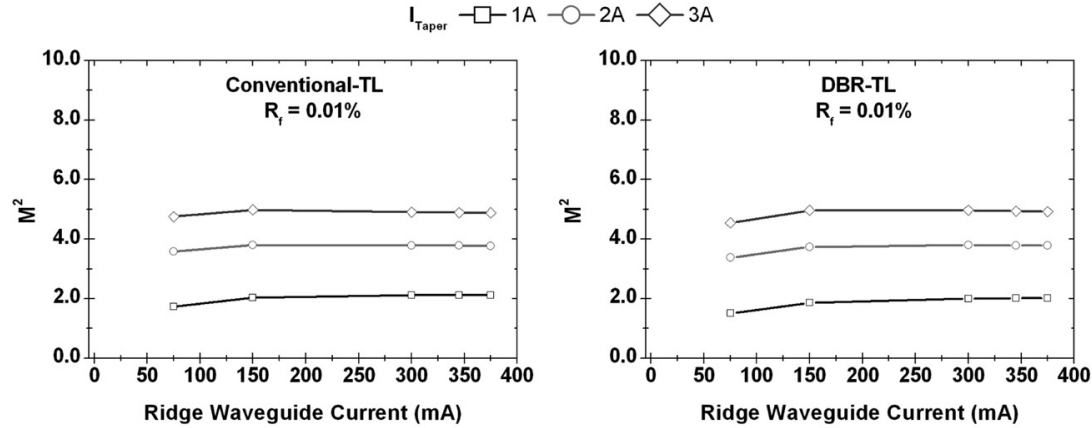

a

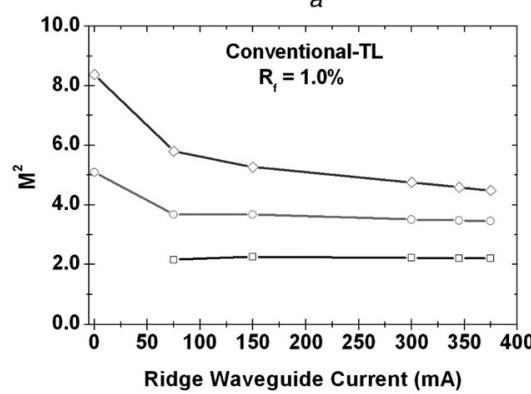

$b$

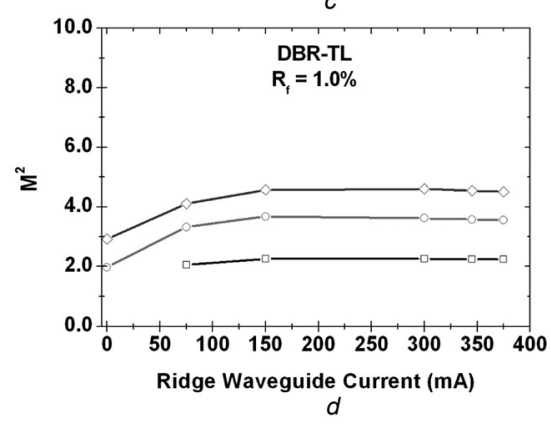

Fig. 7 Evolution of the $M^{2}$ with $R W$ current for the taper currents of 1,2 and $3 \mathrm{~A}$, respectively, at two different values of the front facet reflectivity, $0.01 \%(a, c)$ and $0.1 \%(b, d)$, for the conventional $(a, b)$ and the DBR $(c, d)$ tapered lasers

filtering performance using a RW filter response function defined as the ratio of the forward to backward propagating fields at the RW/taper interface [10]. Fig. 8 shows this filter response function for the front facet reflectivities investigated at a taper current of $3 \mathrm{~A}$. For the conventional tapered laser, a decrease in the ratio of the central lobe to the sidelobes in the filter response is evident as the front facet reflectivity increases (Figs. $8 a$ and $b$ ), particularly at the low RW currents. The small ratio of the central peak to the sidelobes in Fig. $8 b$ is indicative of the deteriorating beam quality (i.e. poor $M^{2}$ value, see Fig. $7 b$ ). In contrast, the RW filter response has a strong central peak for the DBR tapered laser, with good suppression of the side modes for all the values of the front facet reflectivity investigated (Figs. $8 c$ and $d$ ).

\subsection{Influence of the taper angle}

A recent trend has been to use mini-bars with fewer, but higher power emitters. To increase the power of a single
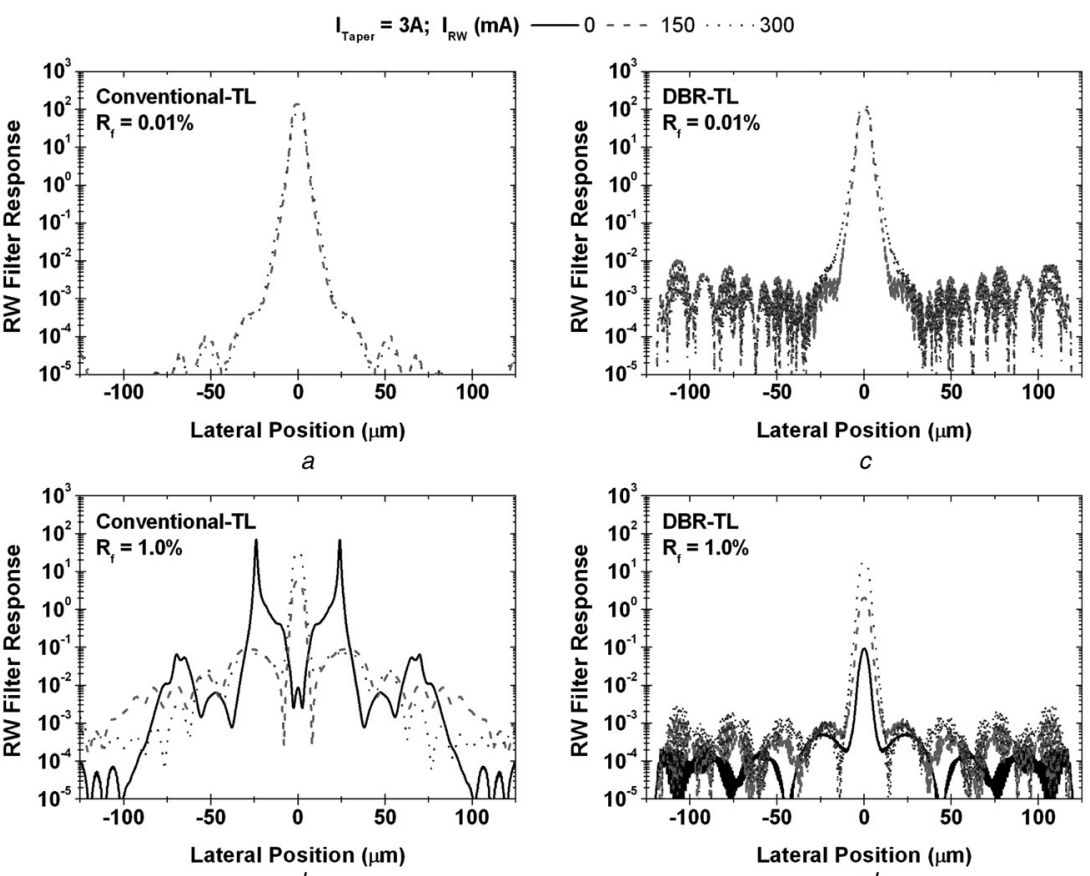

Fig. $8 R W$ filter performance at the RW currents of 0,150 and $300 \mathrm{~mA}$ and a taper current of $3 \mathrm{~A}$ for two different values of the front facet reflectivity, $0.01 \%(a, c)$ and $0.1 \%(b, d)$, for the conventional $(a, b)$ and the DBR $(c, d)$ tapered lasers 


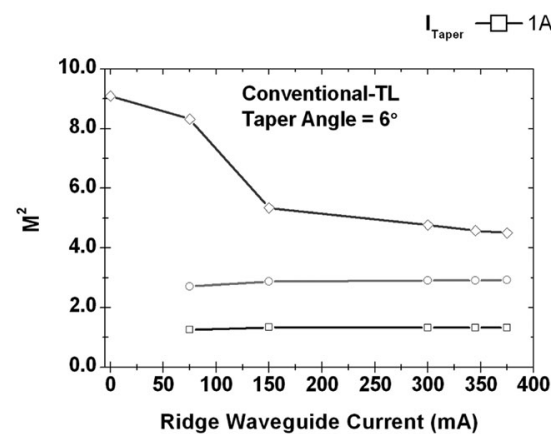

a

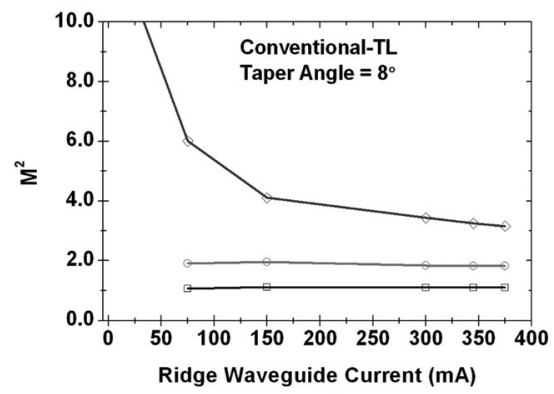

b

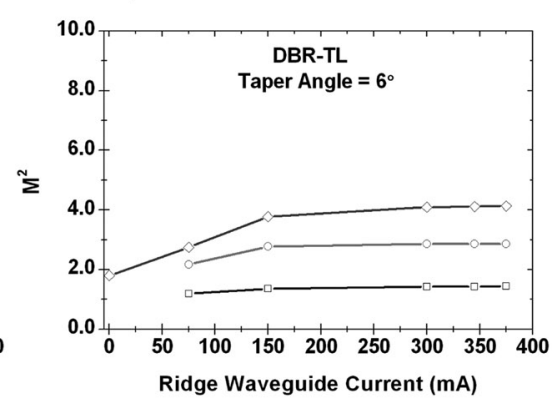

$c$

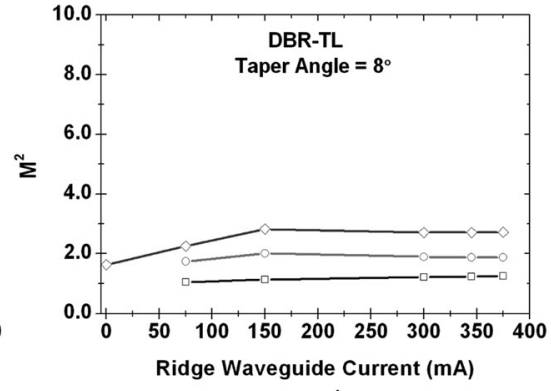

d

Fig. 9 Evolution of $M^{2}$ with the $R W$ current for the taper currents of 1,2 and $3 A$, respectively, for the conventional $(a, b)$ and the DBR (c, d) tapered lasers with taper angles of $6^{\circ}(a, c)$ and $8^{\circ}(b, d)$, respectively

emitter, the gain volume must be increased by using a wider taper angle and/or increasing the length of the taper. In this paper, the length of the taper was constant, but the taper angle was varied to investigate its effect on the beam quality. Two taper angles, $6^{\circ}$ and $8^{\circ}$, respectively, were investigated and compared with the standard device with a $4^{\circ}$ taper angle, for both the conventional and the DBR tapered lasers. For a taper current of 3 A, Fig. 9 shows the evolution of the $M^{2}$ as a function of the RW current for the $6^{\circ}$ and the $8^{\circ}$ taper angles for both the conventional and the DBR tapered lasers. The beam quality degrades with an increasing taper current (accompanied by an increase in the output power) in the conventional tapered lasers (Figs. 9a and $b$ ), particularly at the low RW currents. For example, when the taper angle is greater than $6^{\circ}, M^{2}>8$ for the RW and the taper currents of $0 \mathrm{~mA}$ and $3 \mathrm{~A}$, respectively. The deterioration of the beam quality is due to the mismatch between the free diffraction angle of the field from the RW
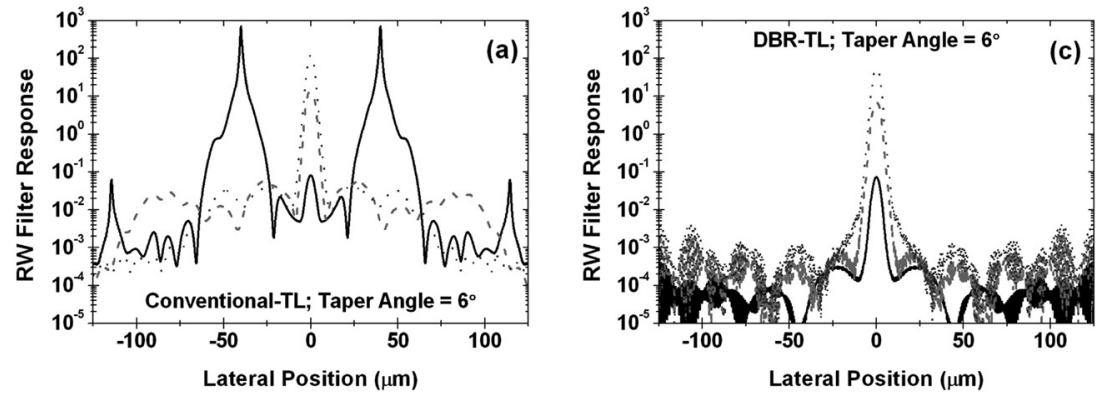

c
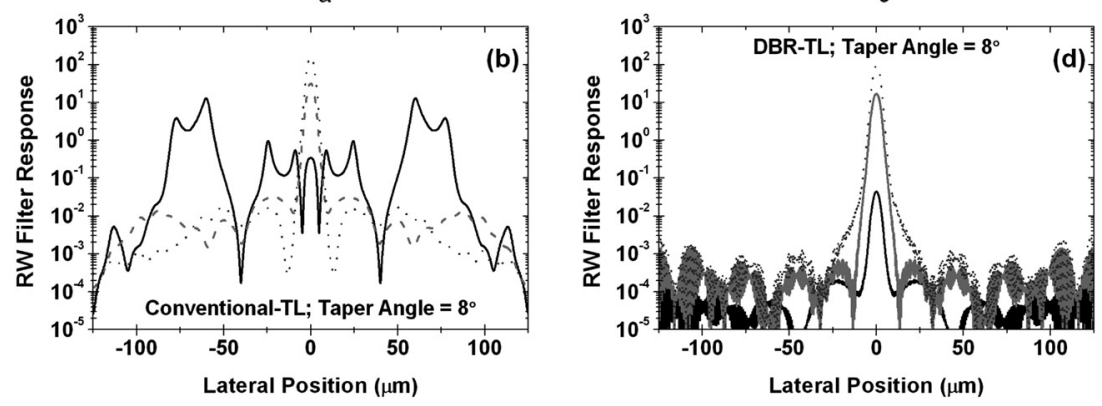

Fig. $10 R W$ filter performance at the $R W$ currents of 0,150 and $300 \mathrm{~mA}$, respectively, and a taper current of $3 \mathrm{~A}$ for the conventional (a, $b$ ) and the DBR $(c, d)$ tapered lasers with taper angles of $6^{\circ}(a, c)$ and $8^{\circ}(b, d)$, respectively 
section and the taper angle. The mismatch excites the higher-order modes in the taper, leading to an increased spatial hole burning and the formation of a carrier-induced lens, which guides the light, forming narrow filaments with a large far-field divergence. In addition, there are significantly higher carrier densities outside the tapered section than in the RW because of carrier spreading. These high carrier densities amplify the backward travelling fields in the electrically unpumped regions alongside the taper. This increases the optical bleaching of the RW section and reduces the mode filtering efficiency. To improve the beam quality for the larger taper angles, it is necessary to optimise the design of the RW in the conventional tapered lasers. In contrast, the spatial mode filtering by the DBR-RW remains effective in the structures with larger taper angles (Figs. $9 c$ and $d$ ). The above observations are confirmed by the RW mode filtering performance (response function) shown in Fig. 10.

\section{Use of a beamspoiler in the conventional tapered laser diodes}

It has been demonstrated that the beam quality in the conventional tapered lasers is generally inferior compared with the DBR tapered lasers because of the poorer RW filter performance of the conventional tapered lasers. The beam quality in the conventional tapered lasers can be improved by using beamspoilers at the RW/taper junction [11] to reflect away the backward propagating beams into the lossy, unpumped regions of the material. The effect of using a beamspoiler in a conventional tapered laser was investigated and compared against the performance of the DBR tapered laser (without a beamspoiler). Fig. 11 shows the near-field patterns and the evolution of the $M^{2}$ as a function of the RW current at fixed taper currents for a conventional tapered laser incorporating a beamspoiler
(Figs. $11 a$ and $b$ ) and a DBR tapered laser that does not incorporate a beamspoiler (Figs. $11 c$ and $d$ ). The beam quality of the conventional tapered laser with a beamspoiler is comparable with that of the DBR tapered laser, which does not have a beamspoiler. Although beamspoilers are relatively easy to fabricate, the DBR tapered lasers offer further advantages in applications that require stable wavelength operation and narrow spectral linewidth, for example, mini-bars.

\section{Conclusion}

Factors affecting the brightness of the two-section, high-power tapered laser diodes were investigated. The propagation of the higher-order modes in the regions adjacent to the RW and their subsequent reflections back along the cavity are shown to be the cause of the sidelobes observed in the near-field patterns. These higher-order modes are excited in the conventional tapered lasers by optical bleaching of the regions outside the RW, especially at the high taper currents and the low RW currents. Similarly, higher front-facet reflectivities and larger taper angles result in an increase in the optical bleaching because of the significant back reflections from the front facet. Higher-order modes are also excited in the larger taper angle devices because of the mismatch between the taper and the RW. The large sidelobes in the near-field lead to a multi-lobed far-field and degraded beam quality in the conventional tapered lasers. The spatial mode filter in the DBR laser improves the performance of the RW filter, since the combined effect of the RW mode filter and the DBR reflector at the back facet suppress the higher-order lateral modes (by allowing them to continue to propagate out through the AR-coated rear facet). An alternative method of improving the beam quality in the conventional tapered laser diodes is the inclusion of a beamspoiler at the $\mathrm{RW} /$ taper junction. It has been shown that the DBR

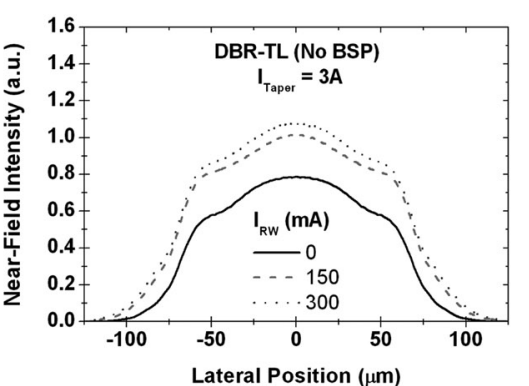

c

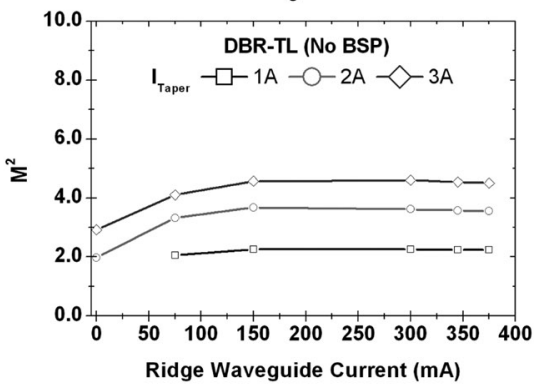

d

Fig. 11 Comparison of the performance of a $a, b$ Conventional tapered laser with a beamspoiler and $c, d$ DBR tapered laser without a beamspoiler

Near-field patterns $(\mathrm{a}, \mathrm{c})$ for the RW currents of 0,150 and $300 \mathrm{~mA}$, respectively, and a taper current of $3 \mathrm{~A}$; evolution of the $M^{2}$ with the RW current for the taper currents of 1,2 and $3 \mathrm{~A}(\mathrm{~b}, \mathrm{~d})$, respectively 


\section{www.ietdl.org}

tapered laser has a superior beam quality for a wider range of operating conditions compared with the conventional tapered lasers, even those employing beamspoilers. In particular, for 1060 applications that require low RW bias currents, the DBR tapered lasers will be an important source as high modulation efficiencies can be achieved (by using the low RW currents) while maintaining a high-power output (at the high taper currents).

1065

\section{Acknowledgments}

The authors gratefully acknowledge the support of the EC FP7 projects $W W W . B R I G H T E R . E U$ (Project no. 035266) and BRIDLE (Project no. 314719).

\section{References}

1 Schulz, W., Poprawe, R.: 'Manufacturing with novel high-power diode lasers', IEEE J. Sel. Top. Quantum Electron., 2000, 6, (4), pp. 696-705

2 Marciante, J.R., Agrawal, G.P.: 'Spatio-temporal characteristics of filamentation in broad-area semiconductor lasers: experimental results', IEEE Photonics Technol. Lett., 1998, 10, (1), pp. 54-56

3 Lichtenstein, N., Manz, Y., Mauron, P., Fily, A., Arlt, S.: 'Single mode emitter array laser bars for high-brightness applications'. Proc. Int. Semiconductor Laser Conf., Matsue-shi, Japan, September 2004, pp. 45-46

4 Wilson, F.J., Lewandowski, J.J., Nayar, B.K et al.: '9.5 W CW output power from high brightness $980 \mathrm{~nm}$ InGaAs/AlGaAs tapered laser arrays', Electron. Lett., 1999, 35, (1), pp. 43-45
5 Sumpf, B., Wenzel, H., Erbert, G.: 'High-power, high-brightness semiconductor tapered diode lasers for the red and infrared spectral range'. Proc. SPIE, 7616, Novel In-Plane Semiconductor Lasers IX, California, USA, February 2010, p. 76161 L

6 Mikulla, M.: 'Tapered high-power, high-brightness diode lasers: design and performance', Top. Appl. Phys., 2000, 78, pp. 265-288

7 Keleman, M.T., Keifer, R., Mikulla, M., et al.: 'High-power, high brightness ridge-waveguide tapered diode lasers at $940 \mathrm{~nm}$ '. Proc. SPIE, 2002, vol. 4648, pp. 75-81

8 Sujecki, S., Borruel, L., Wykes, J., et al:: 'Nonlinear properties of tapered laser cavities', IEEE J. Sel. Top. Quantum Electron., 2003, 9, (3), pp. 823-834

9 Borruel, L., Sujecki, S., Moreno, P., et al.: 'Quasi-3D simulation of high-brightness tapered lasers', IEEE J. Quantum Electron., 2004, 40, (5), pp. 463-472

10 Bull, S., Kaunga-Nyirenda, S.N., Lim, J.J., Hasler, K.-H., Fricke, J., Larkins, E.C.: 'Analysis of the performance of the ridge waveguide filter in high-power tapered laser diodes', Unpublished

11 Walpole, J.N., Kintzer, E.S., Chinn, S.R., Wang, C.A., Missaggia, L.J. 'High-power strained-layer InGaAs/AlGaAs tapered travelling wave amplifier', Appl. Phys. Lett., 1992, 61, (7), pp. 740-742

12 Hasler, K.-H., Sumpf, B., Adamiec, P., et al.: '5-W DBR tapered lasers emitting at $1060 \mathrm{~nm}$ with a narrow spectral linewidth and a nearly diffraction-limited beam quality', IEEE Photonics Technol. Lett., 2008, 20, (19), pp. 1648-1650

13 Lim, J.J., Sujecki, S., Lang, L., et al.: 'Design and simulation of next-generation high-power, high-brightness laser diodes', IEEE J. Sel. Top. Quantum Electron., 2009, 15, (3), pp. 993-1008

14 Lim, J.J., Benson, T.M., Larkins, E.C.: 'Design of wide-emitter single-mode laser diodes', IEEE J. Quantum Electron., 2005, 41, (4), pp. $506-516$ 


\section{www.ietdl.org}

OPTSI20130082

Simeon Newton Kaunga-Nyirenda, Stephen Bull, Jun Jun Lim, Karl-Heinz Hasler, Jörg Fricke, Eric C. Larkins

Q1 Please check and confirm the insertion of main caption for Figs. 3-6. 